\title{
Breast Apocrine Adenoma
}

National Cancer Institute

\section{Source}

National Cancer Institute. Breast Apocrine Adenoma. NCI Thesaurus. Code C40383.

A rare, benign and well circumscribed neoplasm that arises from the breast. It is characterized by the proliferation of epithelial cells with extensive apocrine metaplasia. 